\title{
Iso-geometric shape optimization of magnetic density separators
}

\author{
Dang Manh, Nguyen; Evgrafov, Anton ; Gravesen, Jens; Lahaye, Domenico
}

Published in:

Compel

Link to article, DOI:

10.1108/COMPEL-07-2013-0234

Publication date:

2014

Document Version

Peer reviewed version

Link back to DTU Orbit

Citation (APA):

Dang Manh, N., Evgrafov, A., Gravesen, J., \& Lahaye, D. (2014). Iso-geometric shape optimization of magnetic density separators. Compel, 33(4), 1416-1433. https://doi.org/10.1108/COMPEL-07-2013-0234

\section{General rights}

Copyright and moral rights for the publications made accessible in the public portal are retained by the authors and/or other copyright owners and it is a condition of accessing publications that users recognise and abide by the legal requirements associated with these rights.

- Users may download and print one copy of any publication from the public portal for the purpose of private study or research.

- You may not further distribute the material or use it for any profit-making activity or commercial gain

- You may freely distribute the URL identifying the publication in the public portal

If you believe that this document breaches copyright please contact us providing details, and we will remove access to the work immediately and investigate your claim. 


\title{
Iso-Geometric Shape Optimization of Magnetic Density Separators
}

\author{
June 5, 2014
}

\begin{abstract}
Purpose - The waste recycling industry increasingly relies on magnetic density separators. These devices generate an upward magnetic force in ferro-fluids allowing to separate the immersed particles according to their mass density. Recently, a new separator design has been proposed that significantly reduces the required amount of permanent magnet material. The purpose of this paper is to reduce the undesired end-effects in the upward force that this design generates by altering the shape of the ferromagnetic covers of the individual poles.

Design/methodology/approach - We represent the shape of the ferromagnetic pole covers with B-splines and define a cost functional that measures the non-uniformity in the magnetic force in an area above the poles. We apply an isogeometric shape optimization procedure, which allows us to accurately represent, analyze and optimize the geometry using only a few design variables. The design problem is regularized by imposing constraints that enforce the convexity of the pole cover shapes. It is solved by a non-linear optimization procedure. We validate the implementation of our algorithm using a simplified variant of our design problem with a known analytical solution. The algorithm is subsequently applied to the problem posed.
\end{abstract}

Research limitations/implications - The shape optimization attains its target and yields pole cover shapes that give rise to a magnetic field that is uniform over a larger domain. This increased uniformity is obtained at the cost of a pole cover shape that differs per pole. This limitation has negligible impact on the manufacturing of the separator. The new pole cover shapes, therefore, lead to improved performance of the density separation.

Originality/value - This paper treats the shapes optimization of magnetic density separators systematically and presents new shapes for the ferromagnetic pole covers. Due to the larger uniformity of the generated field, these shapes should enable larger amounts of waste to be processed than the previous design.

Keywords - Magnetic density separation, shape optimization, iso-geometric analysis.

Paper type - Research paper

\section{Introduction}

Magnetic density separators are increasingly being used by the waste recycling industry. The development and usage of these devices is extensively described in 
the monograph [Svoboda (2004)]. The separators considered here exert an upward magnetic force on waste particles immersed in a container with ferro-fluid. As the magnitude of the resultant of the hydrostatic and magnetic buoyancy force is proportional to the mass density of the waste particles, these particles will float at mass density specific heights. If this height is constant across lateral directions in the container, particles of the same mass density can easily be removed from the container.

The requirements imposed on the magnetic field led to the development of permanent magnet arrays specifically designed for magnetic density separators [Rem et al. (2007)] [Muchova et al. (2009)]. Very recently, a design that significantly reduces the amount of costly permanent magnet material was proposed [Lahaye et al. (2011)]. Given its importance in this paper, this design is shown in Figure 1.

Figure 1(a) shows a front view of the Hallbach-type magnet array considered. Only the part of the configuration to the right of the vertical symmetry axis is depicted. The configuration consists of three permanent magnet poles interleaved by ferromagnetic poles mounted on an iron back plate. All magnets are magnetized upward as indicated by the arrows in the figure. Both the magnet and iron poles are covered by iron caps such that the magnet field above the array is suitably shaped. Assuming that the structure is continued periodically, an analytical expression for the pole cover shape has been proposed in [Lahaye et al. (2011)]. This expression will be derived in Subsection 5.1. In case that the array is truncated to finite length however, end effects in the magnetic field do appear as shown in Figure 1(b). This figure shows the magnitude of the simulated magnetic field as a function of the lateral coordinate $x$ at three values of the height coordinate $y$. For the simulation we employ two-dimensional finite element simulations. The occurrence of the end effects shown perturbs the uniformity of the magnetic force above the array and severely limits the deployment of the magnet array for density separation.

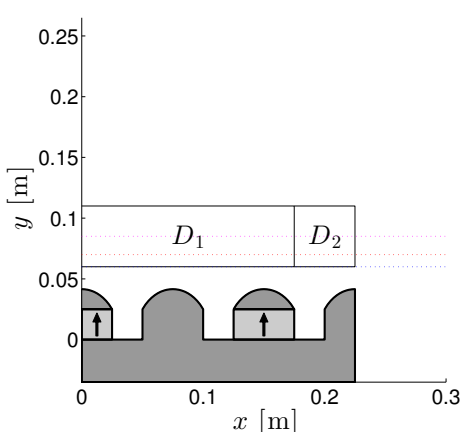

(a)

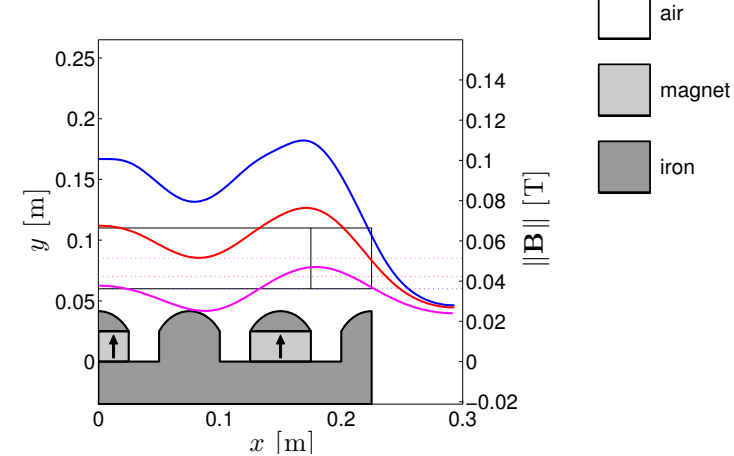

(b)

Figure 1: Front view of the magnet array proposed in [Lahaye et al. (2011)] with the original pole and magnet ordering showing the objective domains $D_{1}$ and $D_{2}$ (left) and the computed $\|\mathbf{B}\|$ at a height of 6,7 and $8.5 \mathrm{~cm}$ above the back plate (right).

Figure 2(a)-(b) shows that the end effects can already be significantly reduced by permuting the position of magnet and iron poles. To goal of this 


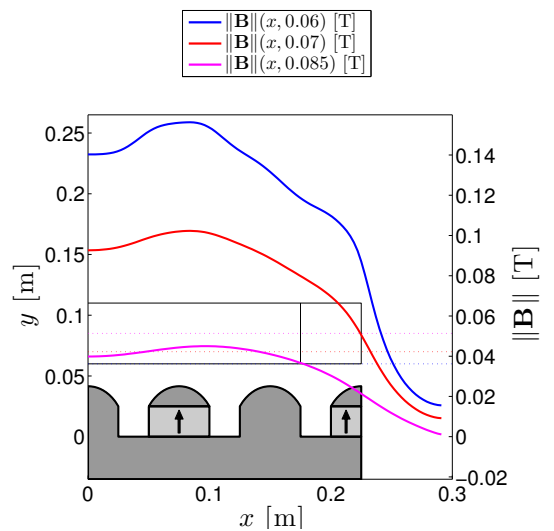

(a)

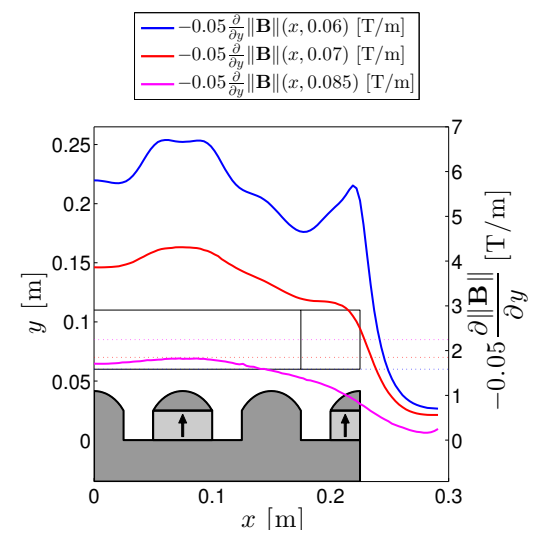

(b)

Figure 2: Front view of the magnet array proposed in [Lahaye et al. (2011)] with the reserved pole and magnet ordering showing the computed $\|\mathbf{B}\|$ (left) and $\frac{\partial\|\mathbf{B}\|}{\partial y}$ (right) at a height of 6,7 and $8.5 \mathrm{~cm}$ above the back plate.

paper is to apply shape optimization of the pole coverings in the configurations shown in Figure 2(a)-(b) in order to further reduce the end-effects. As far as the authors are aware of, the application of shape optimization to magnetic density separators is new. Various approaches to shape optimization of stationary magnetic fields have been proposed by various authors. Without the intention of giving an overview, we here list some references [Tadic et al. (2011)] [Di Barba et al. (2009)] [Kim et al. (2002)] [Saludjian et al. (1998)] [Kim et al. (2002)]. In this paper we propose to use iso-geometric analysis.

Iso-geometric analysis is a modern numerical method for solving partial differential equations [Hughes et al. (2005)] [Cottrel et al. (2009)]. Its name stems from the fact that the same class of functions is used to parametrize the geometry and to solve the differential equation. The computational domain is subdivided into a number of patches such that each patch is the image of the reference element under a parametrization. This parametrization is defined as a linear combination of the tensor products of B-splines. The use of splines allows us to represent complex computational domains with a limited number of design variables. On each patch the basis functions are as the composition of the pullback operator and the tensor product of spline basis function on the reference elements. The use of B-splines as finite element basis functions is described in e.g. the monograph [Höllig (2003)] and the references cited therein. The isogeometric analysis innovative approach of representing both the geometry and the discrete solution using splines allows to continuously vary the basis functions with the underlying shape and avoids the introduction of numerical noise associated with mesh updating procedures associated with traditional FEM procedures. Iso-geometric analysis allows us to compute the sensitivity of the cost functional with respect to changes in the geometry in an efficient and robust manner. This sensitivity defines the descent direction that is used to search the design space using a small number of computationally expensive cost function evaluations. As the geometry is defined in terms of splines, it can easily be imported in available finite element software environments for further analy- 
sis. The advantages of isogeometric analysis for shape optimization are further elaborated in e.g. [Cho et al. (2009)] [Nguyen (2012)] [Nguyen et al. (2012)].

In this paper we apply iso-geometric shape optimization to the magnetic density separators shown in Figure 2(a)-(b). Our goal is to shape the covers of the individual poles in such a way to minimize the non-uniformity of derivative of the magnitude of the magnetic flux in lateral direction in an area above the poles. We introduce a functional that measures this non-uniformity and minimize this functional over two objective domains to investigate the influence of end-effects. Our algorithm produces new shapes that significantly improve the field uniformity and that therefore renders the device much more useful in industrial applications.

This paper is structured as follows: in Section 2 we describe the shape optimization problem we set out to solve. In Section 3 we briefly review the isogeometric analysis and shape optimization technique that we intend to employ. In Section 4 we give more details on the shape representation using B-splines as it is an essential ingredient in the approach that we adopt. In Section 5 the methodology we advocate is tested on a design problem with a known analytical solution and on two versions of the shape optimization problem of the magnetic density separator. In Section 6 finally conclusions are drawn.

\section{Formulation of the Shape Optimization Prob- lem}

In this section we formulate the shape optimization problem of the magnet array by giving details of the magnetic field equation, the cost functional, the design variables and the regularization technique.

The objective of the shape optimization is to find shapes of the covers of the magnet and ferromagnetic poles that yield a magnetic force with a variation in the lateral coordinate that is better suited for the density separation on waste particles immersed in the ferro-fluid in the container placed in the magnetic field. Waste particles in the magnetic field experience the downward gravitational pull, the upward hydrostatic buoyancy force and the upward magnetic force from the ferro-fluid. If the latter is made independent of the lateral $(x-)$ coordinate, the resultant force is laterally invariant as well, and waste particles with the same mass density will float at an laterally invariant height. This facilitates the removal of the different particles from the fluid and renders the device attractive from an industrial point of view. We stress here that unlike other approaches for synthesizing the magnetic field that appeared in the literature, our objective is not to control individual field components, but rather the resulting magnetic force. Computing this force requires computing second order derivatives of the magnetic (either scalar or vector) potential in the post-processing stage of a field analysis.

A ferro-fluid with mass density $\rho_{f}$ and saturation magnetization $M_{f}$ will react to being placed in a spatially varying magnetic field $\mathbf{B}(x, y)$ by a change in its density to its so-called apparent density $\rho_{a p p}$. The latter is proportional to the gradient of the magnitude magnetic field in the $y$-direction $\partial\|\mathbf{B}\| / \partial y$. More 
precisely, we have that [Rosensweig (1987)] [Svoboda (2004)]

$$
\rho_{a p p}=\rho_{f}+\frac{M_{f}}{g} \frac{\partial\|\mathbf{B}\|}{\partial y},
$$

where $g$ is the gravitational constant. The computation of the magnetic force requires evaluating second order derivatives of the (scalar or vector) magnetic potential as is typically the case in magnetic force computation methods using for instance the virtual work on Maxwell stress tensor method. In a finite element analysis, these second order partial derivatives can be evaluated element by element. To guarantee sufficient smoothness of the computed second order derivatives we will use in this work second order approximations unless stated otherwise. A contour plot of $\partial\|\mathbf{B}\| / \partial y$ generated by the design shown in Figure 1 is given in [Nguyen (2012)]. The upward force by the ferro-fluid is proportional its apparent density $\rho_{a p p}$. The condition of the lateral invariance of the force by the ferro-fluid can therefore be expressed as

$$
\frac{\partial^{2}\|\mathbf{B}\|}{\partial x \partial y}=0
$$

Our objective is therefore to enforce this condition, at least approximately, over a region located above the magnet array.

From here on we will only consider the magnet array given in [Lahaye et al. (2011)] with reserved magnet and pole ordering shown in Figure 2. Motivating this choice is the fact that the magnets placed at the extremities allows a better control of the end effects. We will compute the magnetic field generated by the magnet array using a vector potential formulation [Sylvester et al. (1996)] In two-dimensional perpendicular current configurations and in the presence of vertically $(y-)$ magnetized permanent magnets with remanent flux density $\mathbf{B}_{r}=$ $\left(0, B_{r}, 0\right)$, the double curl equation for the vector potential $\mathbf{A}=\left(0,0, A_{z}(x, y)\right)$ reduces to

$$
-\frac{\partial}{\partial x}\left(\frac{1}{\mu} \frac{\partial A_{z}}{\partial x}\right)-\frac{\partial}{\partial y}\left(\frac{1}{\mu} \frac{\partial A_{z}}{\partial y}\right)=\frac{1}{\mu} \frac{\partial B_{r}}{\partial x},
$$

where the relative magnetic permeability $\mu_{r}$ is set to 1000 and to 1 in the iron and permanent magnet domain, respectively. The ferro-fluid is diluted with water to such an extend that its influence on the magnetic field is negligible. The neodymium magnets in our simulations have a remanence of $B_{r}=1.235 \mathrm{~T}$. The field equation is supplied with appropriate insulating and symmetry boundary conditions.

The evaluation of Condition (2), requires the computation of third order derivatives of $A_{z}$. To avoid this order of derivation to appear in the objective function, we replace Condition (2) by the minimization of the dispersion $\mathbb{D}(y)$ of $\partial\|\mathbf{B}\| / \partial y$ in $x$-direction, i.e., we aim at reducing the difference between $\partial\|\mathbf{B}\| / \partial y$ and its average value along horizontal lines in $\Omega_{0}=\left[x_{1}, x_{2}\right] \times\left[y_{1}, y_{2}\right]$. This 
motivates the following definition of the cost functional

$$
\begin{aligned}
I_{0}\left[A_{z} ; \Omega_{0}\right] & =\int_{y_{1}}^{y_{2}} \mathbb{D}(y) d y \\
& =\int_{y_{1}}^{y_{2}}\left[\int_{x_{1}}^{x_{2}}\left(\frac{\partial\|\mathbf{B}\|}{\partial y}-\frac{1}{x_{2}-x_{1}} \int_{x_{1}}^{x_{2}} \frac{\partial\|\mathbf{B}\|}{\partial y} \mathrm{~d} x\right)^{2} \mathrm{~d} x\right] \mathrm{d} y \\
& =\int_{y_{1}}^{y_{2}}\left[\int_{x_{1}}^{x_{2}}\left(\frac{\partial\|\mathbf{B}\|}{\partial y}\right)^{2} \mathrm{~d} x-\frac{1}{x_{2}-x_{1}}\left(\int_{x_{1}}^{x_{2}} \frac{\partial\|\mathbf{B}\|}{\partial y} \mathrm{~d} x\right)^{2}\right] \mathrm{d} y(.4)
\end{aligned}
$$

In this functional (with unit $T^{2}$ ) only derivatives of $A_{z}$ up to order two appear. Numerical experiments in Section 5, in which we will seek to minimize the quantity

$$
\mathcal{I}_{0}\left(A_{z} ; \Omega_{0}\right)=\log _{10}\left[I_{0}\left(A_{z} ; \Omega_{0}\right) / \operatorname{area}\left(\Omega_{0}\right)\right]
$$

will give evidence that the cost functional is indeed appropriately chosen. We will perform the optimization using a gradient-based optimization algorithm that requires the derivative of the cost functional with respect to design variables that define the geometry. The deployment of iso-geometric analysis method is motivated by its ability to compute these derivatives without the inconveniences associated with more traditional finite element approaches.

We will conduct numerical studies for two choices for the objective domain $\Omega_{0}$. We define the subdomains $D_{1}$ and $D_{2}$ shown in Figure 1 by

$$
\begin{aligned}
D_{1} & =[0,0.175] \times[0.06,0.11] \quad[\mathrm{m} \times \mathrm{m}], \\
D_{2} & =[0.175,0.225] \times[0.06,0.11] \quad[\mathrm{m} \times \mathrm{m}],
\end{aligned}
$$

respectively. We set $\Omega_{0}$ equal to the domain $D_{1} \cup D_{2}$ in the first study. In the second we restrict the objective domain to the interior by setting $\Omega_{0}=D_{1}$.

\section{Isogeometric shape optimization}

In this section we briefly describe the iso-geometric analysis (IGA) method for solving the magnetic field equation (3) and for the shape optimization of the magnetic density separator shown in Figure 2(a)-(b). This section consists of four subsections. In the first we describe how the geometry is discretized using B-splines in such a way that the designable boundaries can be represented using a limited number of design variables. In the second subsection we cast the magnetic field equation in a Galerkin variational form and discretize this formulation in space using basis functions defined in terms of the domain parametrization. This choice of the basis functions is the key idea of the IGA method. In the third subsection we give the first order sensitivity equations for changes in the coefficients of the discrete solutions with changes in the design parameters describing the geometry. In the fourth subsection we regularize the shape optimization problem introduced. We refer to [Cottrel et al. (2009)] [Cho et al. (2009)] [Hughes et al. (2005)] [Nguyen (2012)] [Nguyen et al. (2012)] for more details on the material presented in this section. 


\subsection{Geometry Discretization}

The IGA method employs the same basis functions to represent both the geometry and the discrete solution of the field equation. In this way the method is similar to the iso-parametric finite element method. The IGA method however uses a more global parametrization of the geometry than classical finite element methods by decomposing the computation domain into a set of patches $\Omega=\cup_{\alpha} \Omega_{\alpha}$. Such a decomposition for the magnetic density separator shown in Figure 2(a)-(b) into a set of 30 patches is shown in Figure 3. In this figure, the ferromagnetic poles with patch number 2 and 17 are parametrized as a single patch while the magnetic poles with patch number 10, 11, 24 and 25 are parametrized using two patches to allow the ferromagnetic caps to cover the magnets. Patches number 3, 6, 12, 15, 18, 21 and 26 and the remaining patches correspond to the ferromagnetic back plate and the air region, respectively. The top boundary of the ferromagnetic and magnetic poles will be allowed to change during the shape optimization process. The global handling of the geometry by patches will facilitate adopting the discretization to changes in the geometry in the next section.

Given the well-documented versatility of splines for representing complex shapes, the IGA method uses these functions as basis functions. In this work we adopt B-splines. Let $u$ and $v$ denotes the coordinates in the parameter space $[0,1]^{2}$. Let $h$ denote the mesh width of an equidistant mesh on $[0,1]$, and let $i$ and $j$ denote the numbering of the basis functions in the $x$ and $y$ direction, respectively. We will parametrize the patch $\Omega_{\alpha}$ using B-splines of order $p$ and $q$ with knot vectors $\Xi_{u}^{\alpha}$ and $\Xi_{v}^{\alpha}$ denoted by $M_{i}^{\alpha, p}(u)$ and $N_{j}^{\alpha, q}(v)$, respectively. Given the knot vector

$$
\Xi_{u}^{\alpha}=\{\underbrace{0, \ldots, 0}_{p+1 \text { times }}, h, 2 h, \ldots, 1-2 h, 1-h, \underbrace{1, \ldots, 1}_{p+1 \text { times }}\},
$$

the set of splines $M_{i}^{\alpha, p}(u)$ is constructed as a linear combination of products of lower order splines. A similar argument holds for the construction $N_{j}^{\alpha, q}(v)$ given $\Xi_{v}^{\alpha}$. We will expand the discrete solution on a patch using the same B-splines. We will denote the tensor product of splines by $R_{i j}^{\alpha, p q}(u, v)=M_{i}^{\alpha, p}(u) N_{j}^{\alpha, q}(v)$.

Each patch $\Omega_{\alpha}$ is parametrized by a linear combination of tensor products of the geometry splines, i.e., $\mathbf{F}_{\alpha}:[0,1]^{2} \rightarrow \Omega_{\alpha}$ where

$$
\mathbf{F}_{\alpha}(u, v)=\left(x_{\alpha}(u, v), y_{\alpha}(u, v)\right)=\sum_{i=1}^{m} \sum_{j=1}^{n} \mathbf{d}_{i j} R_{i j}^{\alpha, p q}(u, v),
$$

where $\mathbf{d}_{i j}$ are the control points. To highlight the dependence of $\mathbf{F}_{\alpha}(u, v)$ on the control points, we will use the notation $\mathbf{F}_{\alpha}(u, v ; \mathbf{d})$. We use spline degree $p=3=q$ on all patches. We will distinguish between patches whose shape is fixed and variable during the design process. On the latter patches, we will treat the boundary and interior control points separately. To control the shape of the boundary of a design-variable patch, we perform a uniform $h \rightarrow H$ coarsening of the corresponding knot vector (7) to obtain

$$
\widehat{\Xi}_{u}^{\alpha}=\{\underbrace{0, \ldots, 0}_{p+1 \text { times }}, H, 2 H, \ldots, 1-2 H, 1-H, \underbrace{1, \ldots, 1}_{p+1 \text { times }}\},
$$


and designate the $x$ and $y$-coordinates of the corresponding control points as design variables. In this construction knot vectors required for the boundary parametrization of $\mathbf{F}_{\alpha}$ are obtained by inserting points uniformly in the knot vector used to describe shape variations. Consequently, the boundary control points are linear combinations of the design control points. This allows to update of the parametrization of an entire patch to shape variations of its boundary and is the distinct feature of the shape optimization using IGA method. This procedure will be outlined in more details in the next section. Figure 3 illustrates this division in control points for the patches corresponding to the magnet $(\alpha=$ $10,11,24,25)$ and ferromagnetic $(\alpha=2,17)$ poles. The $y$-coordinate of the variable boundary control points of patch number 2, 7, 10 and 24 add up to a total of 23 design variables.

\subsection{Field Equation Discretization}

On each patch the basis functions are defined by composing the inverse of the parametrization $\mathbf{F}_{\alpha}$ (also referred to as the pull-back operator) with the tensor of two analysis splines to obtain $R_{i j}^{\alpha, p q} \circ \mathbf{F}_{\alpha}^{-1}(x, y)$. The discrete approximation $u(x, y)$ to the magnetic vector potential over $\Omega_{\alpha}$ can be expanded in this basis as

$$
u(x, y)=\sum_{i}^{m} \sum_{j}^{n} h_{i j}^{\alpha} R_{i j}^{\alpha, p q} \circ \mathbf{F}_{\alpha}^{-1}(x, y) .
$$

To determine the expansion coefficients $h_{i j}^{\alpha}$, we proceed as in any classical finite element method and cast the magnetic field equation (3) in a Galerkin variational formulation. The resulting integrals over $\Omega_{\alpha}$ can be transformed into integrals over $[0,1]^{2}$

$$
\iint_{\Omega_{\alpha}} f(x, y) d x d y=\int_{0}^{1} \int_{0}^{1} f\left(x_{\alpha}(u, v), y_{\alpha}(u, v)\right) \operatorname{det}\left(\mathbf{J}_{\alpha}\right) d u d v
$$

where $\mathbf{J}_{\alpha}=\partial \mathbf{F}_{\alpha} / \partial(u, v)$ denotes the Jacobian of $\mathbf{F}_{\alpha}$, and evaluated via Gaussian quadrature. The weak form on $\Omega_{\alpha}$ then leads to the system of algebraic equations $\mathbf{K}^{\alpha} \mathbf{h}^{\alpha}=\mathbf{f}^{\alpha}$, where $\mathbf{h}^{\alpha}$ contains the coefficients $h_{i j}^{\alpha}$. The entries of $\mathbf{K}^{\alpha}$ are of the form

$$
\mathbf{K}_{k, \ell}^{\alpha}=\frac{1}{\mu} \int_{0}^{1} \int_{0}^{1}\left(\nabla R_{k} \mathbf{J}_{\alpha}^{-1}\right)^{T}\left(\nabla R_{\ell} \mathbf{J}_{\alpha}^{-1}\right) \operatorname{det} \mathbf{J}_{\alpha} \mathrm{d} u \mathrm{~d} v,
$$

where the indices $k$ and $\ell$ correspond to a lexicografic ordering of the unknowns. Given that patches number 11 and 25 are formed by vertically magnetized magnets of size $h_{m}$ and with magnetization $M_{0}$, the entries of $\mathbf{f}^{\alpha}$ are of the form

$$
\mathbf{f}_{\ell}^{\alpha}= \begin{cases}M_{0} h_{m} \int_{0}^{1}\left(R_{\ell}(1, v)-R_{\ell}(0, v)\right) \mathrm{d} v & \text { if } \alpha=11 \text { or } \alpha=25 \\ 0 & \text { otherwise }\end{cases}
$$

Imposing the continuity of both the domain parametrization and the field solution along the patch boundaries results in linear dependencies of a number of control points and expansion coefficients corresponding to neighbouring patches. These can easily be eliminated from the final system as detailed in 
[Nguyen (2012)]. Collecting the contributions from every patch we obtain a system of linear equations

$$
\mathbf{K} \mathbf{h}=\mathbf{f},
$$

where $\mathbf{h}$ contains the expansion coefficients of the solutions on all patches.
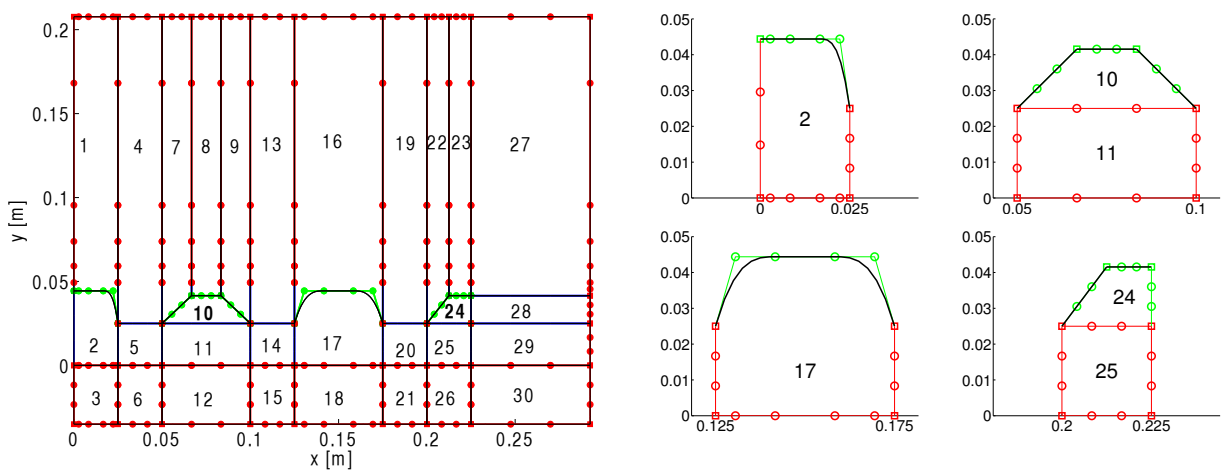

Figure 3: Decomposition of the computational domain $\Omega$ into a set of 30 patches $\Omega_{\alpha}$ (left). Detailed view on the four poles with the boundary control points indicated. (right).

\subsection{Shape sensitivity analysis}

To be able to solve the shape optimization problem by a gradient-based optimization algorithm, the first order sensitivity of the cost functional (4) constrained by the magnetic field equation (3) needs to be computed. To this end, we proceed in a standard way (see e.g. [Lahaye et al. (2012)]). The derivative of the discrete magnetic field solution with respect to the boundary control points $\mathbf{d}_{i j}$ for instance can be found by solving the auxiliary system of linear equations

$$
\mathbf{K} \frac{\partial \mathbf{h}}{\partial \mathbf{d}_{i j}}=\frac{\partial \mathbf{f}}{\partial \mathbf{d}_{i j}}-\frac{\partial \mathbf{K}}{\partial \mathbf{d}_{i j}} \mathbf{h},
$$

obtained by differentiating the system (14). Here we have that $\partial \mathbf{f} / \partial \mathbf{d}_{i j}=\mathbf{0}$. The iso-geometric analysis method allows to compute the derivative $\partial \mathbf{K} / \partial \mathbf{d}_{i j}$ by integration over the reference domain $[0,1]^{2}$. In this way the difficulty of mesh perturbation or updating technigues typically associated with more traditional finite element approaches is alleviated. The optimization problem is solved using a sequential quadratic programming (SQP) algorithm [Nocedal et al. (1999)] implemented in MATLAB's fmincon function using as initial guess the shapes shown in Figure 3.

\subsection{Shape regularization by imposing convexity}

We regularize the shape optimization algorithm by imposing the convexity of the shape of the ferromagnetic pole covers. Let $C(x)$ denote the design curve in the parameter $x$ and assume this curve to be discretized in a set of nodes indexed by $k$. Shape convexity can then be enforced by requiring that the 
second derivative $d^{2} C / d x^{2}$ remains negative. The second order central finite difference discretization of this derivative on the set of nodes results in the set of inequalities

$$
C_{k+1}-2 C_{k}+C_{k-1} \leq 0
$$

that are added to the shape optimization problem. In this way we avoid shapes with strong oscillations or sharp corners.

\section{Domain Parametrization using B-Splines}

In this section we discuss the techniques that we employ to construct a parametrization $\mathbf{F}_{\alpha}$ defined in (8) of a patch $\Omega_{\alpha}$ that is both invertible and of sufficiently high quality. Given the parametrization of the boundary of $\Omega_{\alpha}$ that typically results from a shape updating step in the optimization process, our goal is to compute the control points $\mathbf{d}$ corresponding to the interior control points that satisfy both requirements on $\mathbf{F}_{\alpha}$. The difficulty of this task increases with the geometrical complexity of $\Omega_{\alpha}$. Given that the procedures to find $\mathbf{d}$ have to be applied within each step of an outer optimization algorithm, it is of paramount importance to keep their computational complexity limited.

To ensure regularity of $\mathbf{F}_{\alpha}(u, v)$ we require that given some $\epsilon>0$, the Jacobian $\mathbf{J}_{\alpha}(u, v)$ satisfies $\operatorname{det}\left(\mathbf{J}_{\alpha}(u, v)\right) \geq \epsilon$ for all $(u, v) \in(0,1)^{2}$. We denote by $\operatorname{det}\left[\mathbf{d}_{i j}, \mathbf{d}_{k \ell}\right]$ the determinant of the $2 \times 2$ matrix formed by the $x$ and $y$ coordinates of $\mathbf{d}_{i j}$ and $\mathbf{d}_{k \ell}$. Differentiating (8), we obtain

$$
\begin{aligned}
\operatorname{det}\left(\mathbf{J}_{\alpha}(u, v)\right) & =\sum_{i, j=1}^{m} \sum_{k, \ell=1}^{n} \operatorname{det}\left[\mathbf{d}_{i j}, \mathbf{d}_{k \ell}\right] \frac{\mathrm{d} M_{i}^{\alpha, p}(u)}{\mathrm{d} u} N_{j}^{\alpha, q}(v) M_{k}^{\alpha, p}(u) \frac{\mathrm{d} N_{\ell}^{\alpha, q}(v)}{\mathrm{d} v} \\
& =\sum_{i=1}^{2 m-1} \sum_{j=1}^{2 n-1} c_{i j} M_{i}^{\alpha, 2 p-1}(u) N_{j}^{\alpha, 2 q-1}(v),
\end{aligned}
$$

where $M_{i}^{\alpha, 2 p-1}(u)$ and $N_{j}^{\alpha, 2 q-1}(v)$ are B-splines of order $2 p-1$ and $2 q-1$ over the patch $\Omega_{\alpha}$, respectively, and where to each of the coefficients $c_{i j}$ corresponds a quadratic form determined by the square symmetric matrix $Q_{i j}$ such that [Piegl et al. (1997)]

$$
c_{i j}=\mathbf{d}^{T} Q_{i j} \mathbf{d} .
$$

Given that the B-splines are positive, the regularity of $\mathbf{F}_{\alpha}(u, v)$ can be ensured by imposing that each of the coefficients $c_{i j}$ in (17) is positive.

To ensure that a parametrization $\mathbf{F}_{\alpha}$ is of high quality we require the matrix $\mathbf{g}_{\alpha}=\mathbf{J}_{\alpha}^{T} \mathbf{J}_{\alpha}$ to be well approximated by the identity (see e.g. Corollary 6.4.3 in [Pressley (2010)]). To this end we introduce the Winslow functional $W\left[\mathbf{F}_{\alpha}(\mathbf{d})\right]$ [Knupp et al. (1993)] defined by

$$
W\left[\mathbf{F}_{\alpha}(\mathbf{d})\right]=\iint_{[0,1]^{2}} \mathcal{W}\left[\mathbf{F}_{\alpha}(u, v ; \mathbf{d})\right] d u d v,
$$

where for over $(u, v) \in(0,1)^{2}$ the integrand $\mathcal{W}\left[\mathbf{F}_{\alpha}(u, v ; \mathbf{d})\right]$ is given by

$$
\mathcal{W}\left[\mathbf{F}_{\alpha}(u, v ; \mathbf{d})\right]=\frac{\operatorname{trace}\left(\mathbf{g}_{\alpha}\right)}{\sqrt{\operatorname{det}\left(\mathbf{g}_{\alpha}\right)}}=\frac{\lambda_{1}+\lambda_{2}}{\sqrt{\lambda_{1} \lambda_{2}}}=\frac{\left\|\partial \mathbf{F}_{\alpha} / \partial u\right\|_{2}+\left\|\partial \mathbf{F}_{\alpha} / \partial v\right\|_{2}}{\operatorname{det}\left(\mathbf{J}_{\alpha}\right)},
$$


and where $\lambda_{1}$ and $\lambda_{2}$ denote the eigenvalues of $\mathbf{g}_{\alpha}$. A high quality of $\mathbf{F}_{\alpha}$ then corresponds to as low value of $W\left[\mathbf{F}_{\alpha}(\mathbf{d})\right]$ as possible. Minimizing $W\left[\mathbf{F}_{\alpha}(\mathbf{d})\right]$ over the feasible set of control points $\mathbf{d}$ that yield positive coefficients $c_{i j}$ is however too computationally demanding to be carried out at every step of the outer optimization algorithm. We therefore resort to a two-stage heuristic that is described below.

\subsection{Constructing and Updating the Parametrization}

In the first stage we construct a reference parametrization denoted by $\mathbf{d}_{0}$ by minimizing the Winslow functional (19) over the design space of spline control points $\mathbf{d}$ subject to the constraint that the coefficients $c_{i j}$ defined by (18) remain positive. This optimization problem is solved to local optimality using a nonlinear optimization method, in fact the same as we use in the outer optimization.

In the second stage we update the parametrization $\mathbf{d}$ to the current shape of the patch $\Omega_{\alpha}$ by minimizing the second order Taylor polynomial of the Winslow functional (19) around the point $\mathbf{d}_{0}$. This polynomial can be written as

$$
W\left[\mathbf{F}_{\alpha}(\mathbf{d})\right] \approx W\left[\mathbf{F}_{\alpha}\left(\mathbf{d}_{0}\right)\right]+G\left(\mathbf{d}_{0}\right)\left(\mathbf{d}_{0}-\mathbf{d}\right)+1 / 2\left(\mathbf{d}_{0}-\mathbf{d}\right)^{T} H\left(\mathbf{d}_{0}\right)\left(\mathbf{d}_{0}-\mathbf{d}\right),
$$

where $G\left(\mathbf{d}_{0}\right)$ and $H\left(\mathbf{d}_{0}\right)$ denote the gradient and Hessian of $W\left[\mathbf{F}_{\alpha}\right]$ with respect to the control points $\mathbf{d}$ evaluated in the point $\mathbf{d}_{0}$, respectively. Minimizing this polynomial is then equivalent to solving the linear system of equations

$$
H\left(\mathbf{d}_{0}\right)\left(\mathbf{d}_{0}-\mathbf{d}\right)=-G\left(\mathbf{d}_{0}\right),
$$

resulting in an inexpensive updating formula. At the same time the positivity of coefficients $c_{i j}$ is added as constraints in the outer optimization thus ensuring a valid parametrization throughout the outer optimization. At the end of the parametrization we check if the constraint on any of the coefficients $c_{i j}$ is active. If one of them is, then the reference parametrization $\mathbf{d}_{0}$ is update in a process similar to remeshing in standard finite element methods and we restart the outer parametrization.

\section{$5 \quad$ Numerical experiments}

This section consists of two subsections. In Subsection 5.1 we validate our isogeometric shape optimization algorithm on a synthetic problem for which an analytical expression for the optimal shape is known. In Subsection 5.2 we solve the design problem of the magnet array shown in Figure 2 (a)-(b).

\subsection{Synthetic Problem with Analytical Expression for the Optimal Shape}

In this subsection we show that in the absence of end effects the analytical expression for the optimal shape of the pole cover given in [Lahaye et al. (2011)] can be derived. We first give a concise derivation of the optimal shape that this reference lacks. We subsequently employ this shape to investigate at what rate the difference between the numerically and analytically determined shapes converges to zero as the meshwidth is decreased. We do so for various polynomial orders of the spline approximation. 
To derive the analytical expression for the optimal shape, we consider first the magnetic field generated above an idealized Hallbach magnet array of height $h_{m}$ that extends to infinity in lateral directions. We assume the magnet to be mounted on a ferro-magnetic plate reducing the problem to computing the magnetic field caused by the magnet strip $\left\{(x, y, z) \mid-\infty \leq x \leq \infty,-h_{m} \leq\right.$ $y \leq 0,-\infty \leq z \leq \infty\}$ in the overlying half-space $\{(x, y, z) \mid-\infty \leq x \leq$ $\infty, 0 \leq y,-\infty \leq z \leq \infty\}$. We assumed the magnet to be magnetized in the $y$-direction in such a way that, given some amplitude $M_{0}$ and given some wavelength $\lambda$, the magnet's pre-magnetization vector $\mathbf{M}$ can be written as $\mathbf{M}=\left(0, M_{0} \cos (\pi x / \lambda), 0\right)$. The problem is thus reduced to the coordinates $x$ and $y$. Let $\mu_{r}$ denote the magnet's permeability. To solve the magnetic field problem in the magnet and air region, we solve the Laplace equation for scalar magnetic potential $\phi(x, y)$ supplied with appropriate boundary and interface conditions. The latter are applied on the line $y=0$. We proceed in a similar way to what for example [Cho et al. (2001)] refers to as Type (a) magnet arrays and find that in magnet region the scalar potential varies linearly with $y$. In the air region holds that

$$
\phi(x, y)=C_{1} \cos (\pi x / \lambda) \exp (-\pi y / \lambda),
$$

where $C_{1}$ is an integration constant equal to

$$
C_{1}=\frac{M_{0} h_{m}}{\mu_{r}+\pi h_{m} / \lambda} \exp \left(\frac{\pi h_{m}}{\lambda}\right) .
$$

The magnetic field strength in the region above the magnet is therefore given by

$$
\|\mathbf{B}\|=\mu_{0} \sqrt{(\partial \phi / \partial x)^{2}+(\partial \phi / \partial y)^{2}}=\mu_{0} C_{1} \pi / \lambda \exp (-\pi y / \lambda),
$$

and trivially satisfies Condition (2). In the derivation above, end-effect were neglected.

Hallbach arrays for magnetic density separation have been proposed in literature [Svoboda (2004)]. To reduce the amount of magnetic material used however, a new design in which magnets magnetized in only upward direction and in which the magnet poles are interleaved with iron poles has been proposed in [Lahaye et al. (2011)]. In this design the magnetic field distribution above the poles is brought into the desired shape by covering both the iron and magnetic poles with iron parts as shown in Figure 1. On the air boundary of these ferromagnetic coverings the magnetic flux only has a normal component. The tangential component and therefore the tangential derivative of the scalar magnetic potential is zero on this boundary. This implies that on this boundary the magnetic scalar potential is constant. The optimal shape for this covering is thus known as soon as a scalar potential for the optimal field is known. This optimal scalar potential is given by (23) assuming no end-effects are present. The optimal shape is thus found by setting $\phi(x, y)$ equal to a constant $\phi_{0}$ and making the relationship between $x$ and $y$ explicit to obtain

$$
C_{\text {anal }}(x)=\frac{\lambda}{\pi} \log \left[\cos \left(\frac{\pi x}{\lambda}\right)\right]+C_{2},
$$

where $C_{2}=\lambda / \pi\left(\log C_{1}-\log \phi_{0}\right)$. This curve was used to shape the pole covers in Figure 1. In [Lahaye et al. (2011)] is was verified numerically that a periodic continuation of the configuration shown in Figure 1 does give the a field distribution satisfying Condition (2). 
In the remainder of this subsection we consider a synthetic shape optimization algorithm that has the curve (26) as optimal solution. Our aim is to investigate the rate of convergence of the numerically computed solution to the exact one as function of meshwidth $H$ used to discretize the design curve and of the polynomial degree $p$ of the spline approximation To this end we define, given $y(x)$ a smooth function in $x$ and $\bar{y}=0.2077 \mathrm{~m}$, the computational domain $\Omega_{p}=\{(x, y) \mid-\lambda / 3 \leq x \leq \lambda / 3, \underline{y}(x) \leq y \leq \bar{y}\}$ representing the air domain above a single magnetic pole. On this domain we consider solving the Laplace equation for the scalar potential $\phi$ subject to the exact solution (23) given on the boundary. The goal of the synthetic shape optimization algorithm is to minimize the functional

$$
J_{0}[\phi ; D]=\int_{D}\left(\frac{\partial\|\mathbf{B}\|}{\partial x}\right)^{2} d x d y
$$

(measured in $T^{2}$ ) where $D=[-0.02,0.02] \times[0.06,0.12][\mathrm{m} \times \mathrm{m}]$ by varying the shape of $y(x)$. Motivating this choice for $J_{0}[\phi ; D]$ is that if $\partial_{x}\|\mathbf{B}\|=0$ then automatically $\partial_{x}\left(\partial_{y}\|\mathbf{B}\|\right)=\partial_{y}\left(\partial_{x}\|\mathbf{B}\|\right)=0$ and Condition (2) is satisfied. The evaluation of this cost functional requires second order derivatives of the scalar potential $\phi$. The curve $C_{\text {anal }}(x)$ is given by $(26)$ is the analytical solution to this design problem. Let $C_{\text {opt }}(x)$ denote its approximation computed numerically by the IGA shape optimization algorithm on the discretization defined by the following geometry knot vectors

$$
\boldsymbol{\Xi}_{u}=\{\underbrace{0, \ldots, 0}_{p+1 \text { times }}, \frac{1}{32}, \ldots, \frac{31}{32}, \underbrace{1, \ldots, 1}_{p+1 \text { times }}\} \quad \boldsymbol{\Xi}_{v}=\{\underbrace{0, \ldots, 0}_{q+1 \text { times }}, \frac{1}{5}, \ldots, \frac{4}{5}, \underbrace{1, \ldots, 1}_{q+1 \text { times }}\} .
$$

We compute the scaled L2-norm of the difference between $C_{\text {opt }}(x)$ and $C_{\text {anal }}(x)$ for $H \in\left\{\frac{1}{2}, \frac{1}{4}, \frac{1}{8}, \frac{1}{16}, \frac{1}{24}\right\}, p \in\{2,3,4\}$ and $q=p$. For $p=3$, the afore mentioned choices of $H$ leads to a problem with the number of design variables $N_{d v}$ equal to $N_{d v} \in\{3,5,7,9,17,25\}$. Results are given in Figure 4 .

Figure 4 (a) shows how the scaled L2-norm of error in the computed design curve $\left\|C_{\text {anal }}(x)-C_{\text {opt }}(x)\right\|_{2} /\left\|C_{\text {anal }}(x)\right\|_{2}$ decreases with the number of design variables $N_{d v}$ for the three polynomial degrees. This figure shows that for $p=2$ the error scales as $\mathcal{O}\left(H^{1 / 2}\right)$ and that for $p=3$ and $p=4$ the error scales as $\mathcal{O}\left(H^{p}\right)$. The latter rate is not to be confused with the classical estimate of $\mathcal{O}\left(H^{p+1}\right)$ that holds for errors computed over the entire computational domain that remains fixed during the convergence study. A theoretical framework explaining the observed rate of convergence is in fact not known to the authors. The extension of this synthetic problem in which the cost functional (27) is replaced by (4) as well as the development of the required framework that explains the observed rates is left for further work.

\subsection{Shape Optimization of Pole Covers of Magnetic Den- sity Separators}

Before describing the application of the iso-geometric shape optimization algorithm to the density separator, we evaluate the cost functional (4) on the design proposed in [Lahaye et al. (2011)] with the original and reversed pole ordering. These designs are shown in Figure 1 and Figure 2, respectively. The cost 


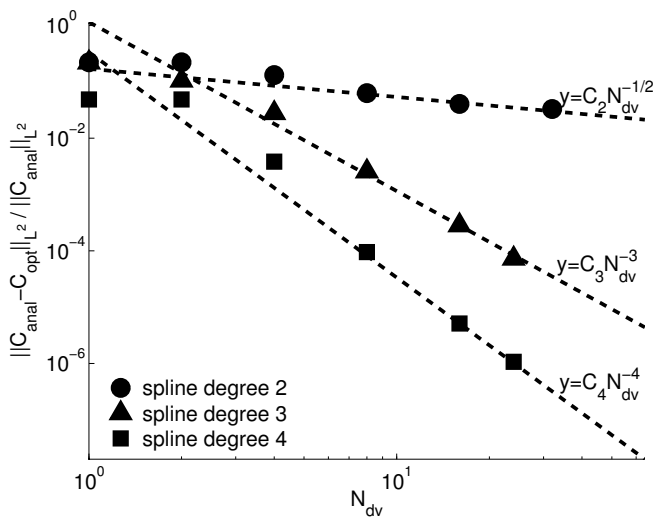

(a)



(b)

Figure 4: Verification of the isogeometric shape optimization algorithm: (a) convergence rate with respect to $h$-refinement for various spline degrees; (b) plot of the absolute error in the found shape for several levels of refinement and $p=q=3$.

function values obtained will serve as reference data in future comparisons. We evaluate the cost functionals $\mathcal{I}_{0}\left(A_{z} ; D_{1}\right)$ and $\mathcal{I}_{0}\left(A_{z} ; D_{2}\right)$, i.e., a measure of the dispersion $\partial\|\mathbf{B}\| / \partial y$ averaged over the domain $D_{1}$ and $D_{2}$ defined by (5). The value for these cost functionals for both designs are given in the first two lines of Table 1. The comparison of these two lines shows that assuring the end pole to be a magnet pole reduces the average dispersion in both $D_{1}$ and $D_{2}$. The numbers shown quantify the statement that switching the magnet-iron pole ordering around is effective in obtaining a more uniform field above the magnet array. To show the effect of the reduction of the dispersion of $\|\mathbf{B}\|$ above the magnet array, we plotted in Figure 2(a) and Figure 2(b) $\|\mathbf{B}\|(x, y)$ and $\partial\|\mathbf{B}\| / \partial y$ along three horizontal lines at height $y=6 \mathrm{~cm}, 7 \mathrm{~cm}$, and $8.5 \mathrm{~cm}$ above the back plate. Figure 2(b) will act as a reference in the qualitative improvement of $\partial\|\mathbf{B}\| / \partial y$ by iso-geometric shape optimization.

In all of the numerical experiments we use the subdivision of the computational domain in patches shown in Figure 3 and a spline approximation of degree $p=3=q$. The third and fourth lines of Table 1 demonstrate that the design of the magnet array can be further improved using iso-geometric shape optimization. These two lines list the average value of the dispersion $\mathcal{I}_{0}\left(A_{z} ; D_{1}\right)$ and $\mathcal{I}_{0}\left(A_{z} ; D_{2}\right)$ over the domain $D_{1}$ and $D_{2}$ for two geometries resulting from a the shape optimization process. The two optimization strategies differ in the choice of the objective domain. The third and fourth line of Table 1 correspond to choosing the objective domain $\Omega_{0}$ defined by (4) equal to $D_{1} \cup D_{2}$ and $D_{1}$, respectively. These variants require 8 and 25 iterations to converge. For both variants, the decrease in the cost function value during the optimization process in shown in Figure 5. A monotone decrease in the cost functional can be observed. The fact that the optimization process continues despite the fact that a close to optimal cost function value has been reached, is likely to be due to the stringent stopping criteria imposed. The third line in Table 1 shows that the shape optimization process is successful in finding a geometry of the pole 
coverings that reduces the cost functional in both $D_{1}$ and $D_{2}$. The fourth line shows that excluding the exterior domain $D_{2}$ from the optimization allows to find a geometry with a lower cost functional in $D_{1}$ at the expense of a higher cost functional over $D_{2}$, demonstrating that the end-effects are harder to control than the interior domain.

In Figure 6(a)-(b) we plotted the geometry resulting from an optimization process with $D_{1} \cup D_{2}$ as objective domain as well as the distribution of $\|\mathbf{B}\|$ and $\partial\|\mathbf{B}\| / \partial y$ over the magnet array. Compared with the initial geometry, the second pole (counting from the left) is lowered and the third pole covering is more asymmetric. The increased uniformity in both $\|\mathbf{B}\|$ and $\partial\|\mathbf{B}\| / \partial y$ can clearly be seen. The compensation for the end-effect causes oscillations in $\partial\|\mathbf{B}\| / \partial y$ with an amplitude that decreases with the height. Further study is required to what extent this limits the applicability of the new shape. We do expect however the impact of these oscillations to be negligibly small in practice. The cover of the second pole is so small that the assumption of a constant permeability might fail. A parameter study in the permeability has however revealed that the obtained field distribution above the magnet array considered has a limited sensitivity in the permeability as long as this permeability remains sufficiently large. This statement could be quantified in a follow-up study.

Figure $6(\mathrm{c})-(\mathrm{d})$ corresponds to $D_{1}$ as objective domain. Compared with the initial geometry, the second pole is lowered and the fourth pole is less rounded. Figure 6(d) shows that by excluding the exterior region from the design close to flat lines for $\partial\|\mathbf{B}\| / \partial y$ can be obtained. This is in stark contrast with the situation shown in Figure 6 (b) and shows that the optimization target set forth in Section can be reached in $D_{1}$. The oscillations in $\partial\|\mathbf{B}\| / \partial y$ in Figure $6(\mathrm{~d})$ are smaller in amplitude than those in Figure 6(b). Figure 6 shows that our designs reduce the end effects significantly. They are therefore promising to apply in realistic industrial applications.

\begin{tabular}{lcccc}
\hline Design & $\Omega_{0}$ & $\#$ it & $\mathcal{I}_{0}\left(A_{z} ; D_{1}\right)$ & $\mathcal{I}_{0}\left(A_{z} ; D_{2}\right)$ \\
\hline original pole ordering in [Lahaye et al. (2011)]: Fig. 1 & - & - & -3.072 & -2.334 \\
reversed pole ordering: Fig. 2 (a) - (b) & - & - & -3.147 & -2.55 \\
IGA optimized: Fig. 6 (a) - (b) & $D_{1} \cup D_{2}$ & 8 & -3.926 & -3.269 \\
IGA optimized: Fig. 6 (c) - (d) & $D_{1}$ & 25 & -4.755 & -2.251 \\
\hline
\end{tabular}

Table 1: Values of the cost functional $\mathcal{I}_{0}$ defined by (5) over the objective domains $D_{1}$ and $D_{2}$ for four designs of magnetic density separators.

\section{Conclusions}

In this paper we improved a recently proposed design of a magnet array used for the hydrostatic separation of waste particles. The improvement was implemented in two stages. In the first stage we reversed the ordering of the iron and permanent magnet poles in such a way that magnets placed at the extremities of the array. This by itself contributes significantly in the reduction of the end-effects in the upward magnetic force. In the second stage we apply shape optimization to the ferromagnetic covers of the individual poles aiming at further reducing the end effects. 


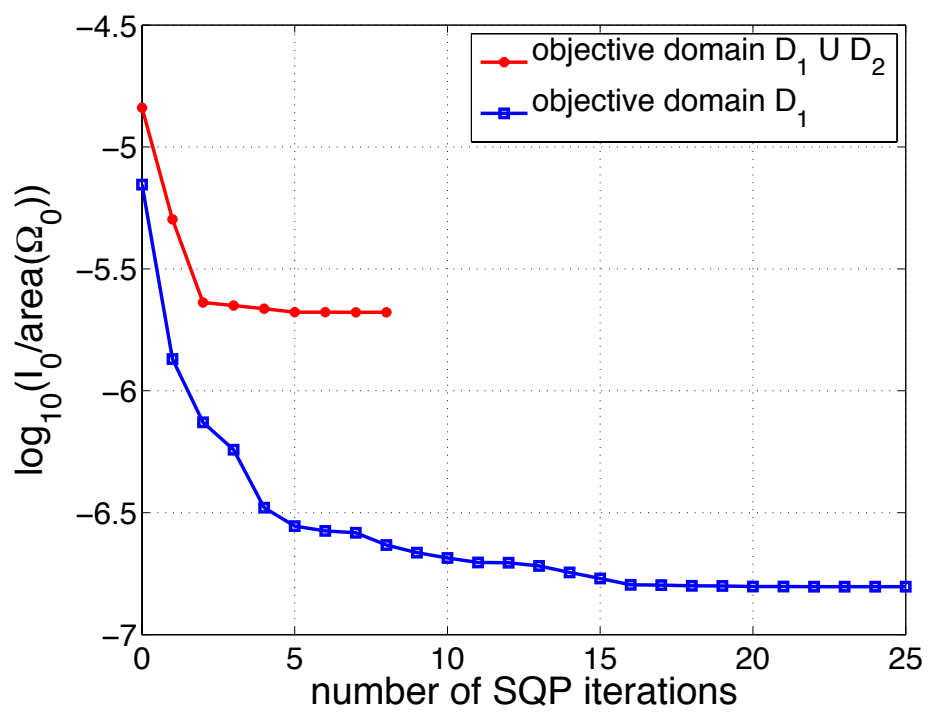

Figure 5: Number of sequential quadratic programming (SQP) iterations to solve the optimization problem with objective domain $\Omega_{0}=D_{1} \cup D_{2}$ and $\Omega_{0}=$ $D_{1}$.

We developed and applied an iso-geometric analysis-based shape optimization algorithm. The distinct feature of this algorithm is the representation of the both the geometry and the discrete solution of the field equation using B-splines. This allows to globally update the discretization with small perturbations in the geometry and therefore to accurately compute the first order sensitivity of the cost functional in the design variables. These derivatives are subsequently used to determine the descent direction in gradient-based optimization algorithms. The latter are well-known to outperform derivative-free optimization methods.

Prior to applying the iso-geometric shape optimization algorithm to the problem of the density separator, we successfully validated our implementation using a design problem with a known analytical solution. Next we considered two variants of the problem of the magnet density separator that differ in the choice of the objective domain. Numerical results for the variant with the largest objective domain show that the end-effect in the upward magnetic force can be substantially reduced at the slight disadvantage of obtaining a pole cover shape that differs per pole. Results for the objective domain that excludes the region above the magnets placed at the extremities show that the magnetic force can be made even more uniform if the region above the end poles are neglected. This force uniformity will cause the waste particles to float at about the same height and facilitate their removal from the container. The design problem was thus solved up to the specifications resulting in new shapes for the ferromagnetic parts covering the poles in the array. The goal of this paper was to contribute to the future developments of magnetic density separators. 


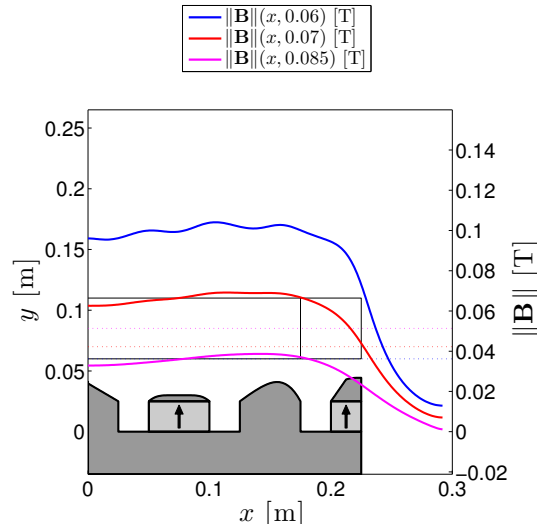

(a)



(c)



(b)

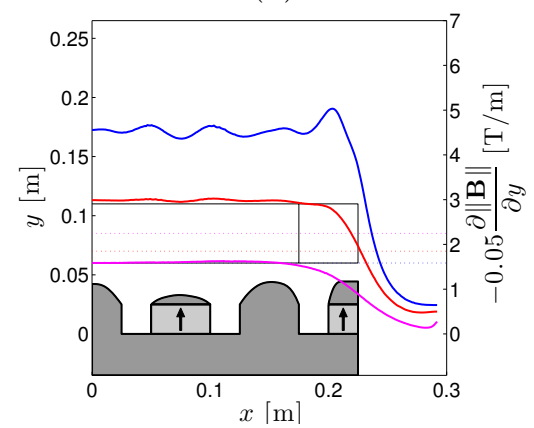

(d)

Figure 6: The graphs of $\|\mathbf{B}\|$ (left) and $\frac{\partial\|\mathbf{B}\|}{\partial y}$ (right) superimposed on the optimized designs. On top the design that corresponds to the objective domain $\Omega_{0}=D_{1} \cup D_{2}$. At the bottom the design that corresponds to $\Omega_{0}=D_{1}$.

\section{Acknowledgment}

Nguyen Dang Manh gratefully acknowledges the financial support from the European Community's Seventh Framework Programme FP7/2007-2013 under grant agreement number PITN-GA-2008-214584 (SAGA). Domenico Lahaye gratefully acknowledges Henk Polinder and Peter Rem for the many fruitful discussions during the course of this work. The authors thank the four anonymous referees for their valuable comments on the previous version of this work.

\section{References}

[Rem et al. (2007)] E. J. Bakker, S. P. M. Berkhout, and P. C. Rem. Method and device for separating solid particles on the basis of a difference in density. Patent EP 1800753/B1.

[Cho et al. (2001)] H. Cho, C. Im, and K. Jung. Magnetic field analysis of 2-D permanent magnet array for planar motor. Magnetics, IEEE Transactions on, 37(5):3762-3766, Sep 2001. 
[Cho et al. (2009)] S. Cho and S. Ha. Isogeometric shape design optimization: exact geometry and enhanced sensitivity. Struct. Multidiscip. Optim., 38(1):53-70, 2009.

[Cottrel et al. (2009)] J. A. Cottrell, T. J. R. Hughes, and Y. Bazilevs. Isogeometric Analysis: Toward Integration of CAD and FEA. J. Wiley., West Sussex, 2009.

[Di Barba et al. (2009)] P. Di Barba and M.E. Mognaschi. Industrial design with multiple criteria: Shape optimization of a permanent-magnet generator. Magnetics, IEEE Transactions on, 45(3):1482 -1485, Mar 2009.

[Höllig (2003)] K. Höllig. Finite Element Methods with B-Splines. Number 26 in Frontiers in Applied Mathematics. SIAM, 2003.

[Hughes et al. (2005)] T.J.R. Hughes, J.A. Cottrell, and Y. Bazilevs. Isogeometric analysis: CAD, finite elements, NURBS, exact geometry and mesh refinement. Comput. Methods Appl. Mech. Engrg., 194(39-41):4135-4195, 2005.

[Kim et al. (2002)] D. Kim, S. Lee, I. Park, and J. Lee. Derivation of a general sensitivity formula for shape optimization of 2-D magnetostatic systems by continuum approach. Magnetics, IEEE Transactions on, 38(2):1125 -1128, Mar 2002.

[Kim et al. (2002)] D.H. Kim, J.K. Sykulski, and D.A. Lowther. The implications of the use of composite materials in electromagnetic device topology and shape optimization. Magnetics, IEEE Transactions on, 45(3):1154 1157, Mar 2009.

[Knupp et al. (1993)] P. Knupp and S. Steinberg. Fundamentals of Grid Generation. CRC Press, Boca Ranton, 1993.

[Lahaye et al. (2011)] D. Lahaye, H. Polinder, and P. Rem. Magnet designs for magnetic density separation of polymers. The Journal of Solid Waste Technology and Management, 26:977-983, 2011.

[Lahaye et al. (2012)] D. Lahaye and W. Mulckhuyse, (2012) Adjoint sensitivity in PDE constrained least squares problems as a multiphysics problem. COMPEL, 31(3):895-903, 2012.

[Muchova et al. (2009)] Muchova L., E. J. Bakker, and P. Rem. Precious Metals in Municipal Solid Waste Incineration Bottom Ash. Water Air Soil Pollution, 9(1-2):107-116, 2009.

[Nguyen (2012)] D. Manh Nguyen. Isogemetric Analysis and Shape Optimization in Electromagnetics. PhD thesis, Technical University of Denmark, 2012.

[Nguyen et al. (2012)] D.M. Nguyen, A. Evgrafov, and J. Gravesen. Isogeometric shape optimization for electromagnetic scattering problems. Progress in Electromagnetics Research B, 46:117-146, 2012.

[Nocedal et al. (1999)] J. Nocedal and S. J. Wright. Numerical Optimization. Springer Series in Operations Research. Springer, 1999. 
[Piegl et al. (1997)] L. Piegl and W. Tiller. The NURBS book. Springer-Verlag, New York, NY, USA, second edition, 1997.

[Pressley (2010)] A. N. Pressley. Elementary Differential Geometry. Springer, second edition, 2010.

[Rosensweig (1987)] R. E. Rosensweig. Magnetic fluids. Ann. Rev., 19:437-463, 1987.

[Saludjian et al. (1998)] L. Saludjian, J.L. Coulomb, and A. Izabelle. Genetic algorithm and Taylor development of the finite element solution for shape optimization of electromagnetic devices. Magnetics, IEEE Transactions on, 34(5):2841-2844, Sep 1998.

[Svoboda (2004)] J. Svoboda. Magnetic Techniques for the Treatment of Materials. Kluwer, Dordrecht, The Netherlands, 2004.

[Sylvester et al. (1996)] P. P. Sylvester and R. L. Ferrari. Finite Elements for Electrical Engineers. Cambridge University Press, New York, third edition, 1996.

[Tadic et al. (2011)] T. Tadic and B.G. Fallone. Three-dimensional nonaxisymmetric pole piece shape optimization for biplanar permanent-magnet MRI systems. Magnetics, IEEE Transactions on, 47(1):231 -238, Jan. 2011. 